\title{
AVALIAÇÃO DE MISTURAS EM TANQUE DE IMAZETHAPYR COM OUTROS HERBICIDAS PARA O CONTROLE DE Bidens pilosa E Euphorbia heteropliylla EM SOJA
}

\author{
Jamil Constantin ${ }^{1}$, Rubem S. de Oliveira Jr. ${ }^{1}$ e Cleber Daniel G. Maciel ${ }^{2}$ \\ 1 Universidade Estadual de Maringá. Departamento de Agronomia. Avenida Colombo, 5790. Maringá, PR 87020-900 \\ ${ }^{2}$ UNESP. Botucatu, SP
}

\section{RESUMO}

Esse estudo teve por objetivo avaliar a seletividade para soja e eficiência de misturas em tanque de imazethapyr com outros latifolicidas. Os tratamentos avaliados foram imazethapyr $(100 \mathrm{~g} / \mathrm{ha})$, imazethapyr + chlorimuron-ethyl $(50+10,50+12,5 \mathrm{e} 60+10$ $\mathrm{g} / \mathrm{ha})$, jmazethapyr + lactofen $(50+96,50+120,60+96,60+120$ e $12,5+120 \mathrm{~g} / \mathrm{ha})$, imazethapyr + oxasulfuron $(50+37,5 \mathrm{e}$ $60+37,5 \mathrm{~g} / \mathrm{ha})$, além de chlorimuron-ethyl + lactofen $(12,5+120 \mathrm{~g} / \mathrm{ha})$, oxasulfuron (45 g/ha), chlorimuron-ethyl (15 g/ha), lactofen (168 g/ha), e testemunhas capinada e sem capina. Todos os herbicidas foram aplicados em pós-emergência, quando a soja encontrava-se no estádio V4 a V5 (3 a 4 folhas trifolioladas). As plantas daninhas presentes, densidades e estádios de desenvolvimento na área experimental eram Bidens pilosa (179 plantas $/ \mathrm{m}^{2}, 2$ a 4 folhas) e Euphorbia heterophylla (63 plantas $/ \mathrm{m}^{2}, 4$ a 5 folhas). As misturas de imazethapyr com chlorimuron-ethyl, lactofen e oxasulfuron foram excelentes no controle de ambas espécies, não evidenciando diferenças significativas entre as diferentes misturas ou entre doses de uma mesma mistura. Em relação á E. heterophylla, as misturas contendo imazethapyr foram superiores a chlorimuron-ethy $1+1$ actofen, e ás aplicações isoladas de chlorimuron-ethyl e lactofen. As misturas com imazethapyr apresentaram um efeito residual até 21 dias após a aplicação para E. heterophylla. Nenhum tratamento com herbicidas afetou o desenvolvimento ou produtividade da soja.

Palavras-chave: chlorimuron-ethyl, lactofen, oxasulturon.

\section{ABSTRACT \\ Evaluation of tank mixtures of imazethapyr with other herbicides for Bidens pilosa and Euphorbia heterophylla control in soybean}

This study was intended to evaluate the selectivity to soybean and efficiency to control Bidens pilosa and Euphorbia heterophylla of tank mixtures of imazethapyr and other broadleaf herbicides. Treatments included imazethapyr (100 g/ha), imazethapyr + chlorimuron-ethyl $(50+10,50+12.5$ and $60+10 \mathrm{~g} / \mathrm{ha})$, imazethapyr + lactofen $(50+96,50+120,60+96,60+120$ and $12.5+120 \mathrm{~g} / \mathrm{ha})$, imazethapyr+oxasulfuron $(50+37.5$ and $60+37.5 \mathrm{~g} / \mathrm{ha})$, chlorimuron-ethyl + lactofen $(12.5+120 \mathrm{~g} / \mathrm{ha})$, oxasulfuron (45 g/ha), chlorimuron-ethyl $(15 \mathrm{~g} / \mathrm{ha})$, lactofen $(168 \mathrm{~g} / \mathrm{ha})$, and weedy and weed free checks. All combinations of herbicides were applied postemergence, at V4-V5 soybean growth stage (3 to 4 composite leaves). Weeds, development stage and population density in experimental area were: Bidens pilosa ( 2 to 4 leaves and 179 plants $/ \mathrm{m}^{2}$ ) and Euphorbia heterophylla (4 to 5 leaves and 63 plants $/ \mathrm{m}^{2}$ ). Tank mixtures of imazethapyr with chlorimuron-ethyl, lactofen and oxasulfuron were bighly eificient for both weeds, providing $>97 \%$ control at pre-harvest evaluation, with no significant diferences among different mixtures or among different rates within each mixture. In relation to E. heterophylla, mixtures containing imazethapyr were more efflcient than both chlorimuron-ethyl+lactofen, and isolated use of chlorimuron-ethyl or lactofen. Mixtures containing imazethapyr provided a residual effect up to 21 days afier application for E. heterophylla. No chemical treatment affected soybean development or productivity.

Key-words: chlorimuron-ethyl, lactofen, oxasulfuron. 


\section{INTRODUÇÃO}

Com uma área anual plantada estimada em 12,6 milhões de hectares, a soja é uma das culturas de maior expressão do Brasil, tornando o país o maior exportador de farelo de soja e o segundo maior na produção de grãos e de óleo de soja no mundo (Agrianual, 1999).

Devido à relevância da cultura, existe a necessidade constante de pesquisas para aumentar a eficiência na produção desta oleaginosa, visando o aumento na competitividade do país internacionalmente. Estudos sobre a interferência e métodos de controle de plantas daninhas são de vital importância para que se aumente a produção e a qualidade da soja brasileira. Lorenzi (1991), Karan et al. (1993a; 1993b) e Spadotto \& Luchiari (1993) citam diversas plantas daninhas como problemáticas para a cultura da soja. Dentre elas, o picão-preto (Bidens pilosa) e o amendoim-bravo (Euphorbia heterophylla) são relatados como plantas daninhas comuns na cultura e, conforme sua infestação, exigem controle para que não interfiram negativamente no desenvolvimento da soja.

O uso de herbicidas em pós-emergência é comum no controle de plantas daninhas em soja. A mistura de herbicidas, principalmente os latifolicidas, está se tornando uma prática cada vez mais utilizada, uma vez que essas misturas têm demonstrado um aumento do espectro de ação e, em alguns casos, uma maior eficiência, devido ao efeito sinergístico. Considera-se que a mistura de herbicidas seja também uma prática benéfica na prevenção do aparecimento de plantas daninhas resistentes a herbicidas (Vargas et al., 1999). Tem sido demonstrado que as misturas de tanque contendo herbicidas do grupo das imidazolinonas podem apresentar grande eficiência no controle de B. pilosa (Laca-Buendia \& Rafael, 1 999a, 1 999b) e E. hetrophylla (Gazziero et al., 1999; Melhorança, 1999; Melhorança \& Pereira, 1999; Vidal, 1999). Os herbicidas bentazon, fomesafen, chlorimuron-ethyl, flumiclorac-pentil, acifluorfen-sódico, lactofen, imazamox e imazethapyr são os mais utilizados para o controle de folhas largas em pós-emergência na cultura da soja, isolados ou em mistura em tanque entre si. O oxasulfuron é um novo herbicida com espectro de ação latifolicida, em fase de desenvolvimento para a cultura da soja, e, teoricamente, seria possível sua mistura com os demais.

O presente trabalho tem como objetivos avaliar a eficiência de mistura em tanque de imazethapyr com outros latifolicidas para o controle de plantas daninhas de folhas largas, assim como a fitotoxicidade destas misturas quando aplicadas em pós-emergência na cultura da soja.

\section{MATERIAL E MÉTODOS}

O ensaio foi instalado na Fazenda Onça Parda, no município de Campo Mourão, PR. A semeadura direta da soja foi efetuada em 01/11/96, utilizando-se o cultivar BR16 com espaçamento de $0,45 \mathrm{~m}$ entre linhas e 18 semente sim linear. A adubação de plantio foi de $227 \mathrm{~kg} /$ ha do formulado 00-25-25. Os demais tratos culturais foram feitos segundo as recomendações da Embrapa (1996).

A aplicação em pós-emergência foi realizada em 03/ 12/96, quando a soja encontrava-se com 3 a 4 trifólios. Utilizou-se um pulverizador costal a base de $\mathrm{CO}_{2}$, com bicos leque 110 -SF-02, pressão de $2,0 \mathrm{~kg} / \mathrm{cm}^{2}$, proporcionando um volume de calda de 200 1/ha. Por ocasião da aplicação dos produtos o solo estava úmido, a temperatura do ar era de $24^{\circ} \mathrm{C}$ e a umidade relativa de $80 \%$, céu parcialmente nublado e velocidade do vento de $2,7 \mathrm{~km} / \mathrm{h}$.

As plantas daninhas presentes, determinadas na área da testemunha sem capina, eram constituídas de 179 plantas $/ \mathrm{m}^{2}$ de picão-preto (Bidens pilosa) e 63 plantas $/ \mathrm{m}^{2}$ de amendoim-bravo (Euphorbia heterophylla). No momento da aplicação, o picão-preto estava com 2 a 4 folhas definitivas e o amendoim-bravo com 4 a 5 folhas definitivas.

Foram avaliadas a percentagem de controle (0-100\%) aos 7, 14, 21 e 42 dias após a aplicação (D.A.A.) e na précolheita, a fitotoxicidade (Escala E.W.R.C.) aos 7, 14, 21 e 42 D.A.A., e o número de plantas de soja/3 m na pré-colheita, além da produtividade da cultura.

O delineamento experimental foi o de blocos casualizados com 16 tratamentos e 4 repetições, sendo as parcelas de $2 \mathrm{~m}$ x $10 \mathrm{~m}\left(20 \mathrm{~m}^{2}\right)$. Na tabela 1 encontram-se os tratamentos, modalidade de aplicação, formulação e doses utilizadas. Os dados foram submetidos á análise de variância e as médias foram comparadas pelo teste Tukey a $5 \%$ de probabilidade.

\section{RESULTADOS E DISCUSSÃO}

Na tabela 2 encontram-se os dados de controle de Bidens pilosa. Observa-se que todos os tratamentos com herbicidas resultaram em excelente nível de controle (>92\%) desde o inicio das avaliações até a pré-colheita. Não foram observadas diferenças expressivas entre as diversas misturas com imazethapyr, nem com relação às demais misturas sem imazethapyr. Não foi possível avaliar o efeito residual dos herbicidas aplicados em pós-emergência, uma vez que não houve reinfestação desta espécie.

As percentagens de controle de Euphorbia heterophylla são apresentadas na Tabela 3. Aos 7 dias após as aplicações (D.A.A.) obteve-se controle eficiente apenas nos tratamentos onde o lactofen estava presente, isolado ou em mistura, possivelmente em função do efeito de contato (mais rápido, portanto) deste produto em relação aos demais, todos sistêmicos. Aos 7 D.A.A. não houve diferença significativa entre as diferentes misturas e tratamentos tradicional- 
Tabela 1. Tratamentos, formulação e doses utilizadas em pós-emergência. Campo Mourão-PR, 1996/97.

\begin{tabular}{lcccc}
\hline \multirow{2}{*}{ Tratamento $^{1}$} & \multicolumn{2}{c}{ Formulação } & \multicolumn{2}{c}{ Dose } \\
\cline { 2 - 5 } & \multicolumn{1}{c}{ Tipo } & Concentração & (g i.a./ha) & (g ou l p.c./ha) \\
\hline Testemunha sem capina & - & - & - & - \\
Testemunha capinada & - & - & - & - \\
Imazethapyr+chlorimuron & Sol.Aq.C.+G.D.A & $100 \mathrm{~g} / 1+250 \mathrm{~g} / \mathrm{kg}$ & $50,0+10,0$ & $\mathrm{O}, 501+40,0 \mathrm{~g}$ \\
Imazethapyr+chlorimuron & Sol. Aq. C.+G.D.A. & $100 \mathrm{~g} / 1+250 \mathrm{~g} / \mathrm{kg}$ & $50,0+12,5$ & $0,501++50,0 \mathrm{~g}$ \\
Imazethapyr+chlorimuron & Sol. Aq. C.+G.D.A. & $100 \mathrm{~g} / 1+250 \mathrm{~g} / \mathrm{kg}$ & $60,0+10,0$ & $0,601+40,0 \mathrm{~g}$ \\
Imazethapyr+lactofen & Sol. Aq. C.+C.E. & $100 \mathrm{~g} / 1+240 \mathrm{~g} / 1$ & $50,0+96,0$ & $0,501+0,401$ \\
Imazethapyr+lactofen & Sol. Aq. C.+C.E. & $100 \mathrm{~g} / 1+240 \mathrm{~g} / 1$ & $50,0+120,0$ & $0,501+0,501$ \\
Imazethapyr+ lactofen & Sol. Aq. C.+C.E. & $100 \mathrm{~g} / 1+240 \mathrm{~g} / 1$ & $60,0+96,0$ & $0,601+0,401$ \\
Imazethapyr+ lactofen & Sol. Aq. C.+C. E & $100 \mathrm{~g} / 1+240 \mathrm{~g} / 1$ & $60,0+120,0$ & $0,601+0,501$ \\
Imazethapyr+oxasulfuron & Sol. Aq. C.+D.G & $100 \mathrm{~g} / 1+750 \mathrm{~g} / \mathrm{kg}$ & $50,0+37,5$ & $0,501+50,0 \mathrm{~g}$ \\
Imazethapyr+oxasulfuron & Sol. Aq. C.+D.G. & $100 \mathrm{~g} / 1+750 \mathrm{~g} / \mathrm{kg}$ & $60,0+37,5$ & $0,601+50,0 \mathrm{~g}$ \\
Oxasulfuron ${ }^{2}$ & D.G. & $750 \mathrm{~g} / \mathrm{kg}$ & 45,0 & $60,0 \mathrm{~g}$ \\
Chlorimuron+lactofen & G.D.A.+C.E & $250 \mathrm{~g} / \mathrm{kg}+240 \mathrm{~g} / 1$ & $12,5+120,0$ & $50,0 \mathrm{~g}+0,501$ \\
Imazethapyr & Sol. Aq. C & $100 \mathrm{~g} / 1$ & 100,0 & 1,01 \\
Chlorimuron ${ }^{3}$ & G.D.A. & $250 \mathrm{~g} / \mathrm{kg}$ & 15,0 & $60,0 \mathrm{~g}$ \\
Lactofen & C.E & $240 \mathrm{~g} / 1$ & 168,0 & 0,701
\end{tabular}

' Correspondência entre ingredientes ativos e produtos comeciais: imazethapyr (Pivot), chlorimuron- ethyl (Classic), lactofen (Cobra), oxasulfuron (Chart).

${ }^{2}$ No tratamento com oxasulfuron isolado adicionou-se o espalhante adesivo Cicol a 0,5\% v./v. Cicol: $900 \mathrm{~g}$ de nonil fenoxi (etilenoxi) etanol por litro.

${ }^{3}$ No tratamento com chlorymuron isolado adicionou-se o óleo mineral Assist a $0,5 \% \mathbf{v} . / \mathbf{v}$.

Tabela 2. Efeitos dos tratamentos no controle de Bídens pilosa na cultura da soja. Campo Mourão-PR, 1996/97.

\begin{tabular}{|c|c|c|c|c|c|c|}
\hline \multirow{3}{*}{ Tratamento } & \multirow{3}{*}{$\begin{array}{c}\text { Dose } \\
\text { (g/ha) }\end{array}$} & \multicolumn{5}{|c|}{ Controle $(\%)$} \\
\hline & & \multicolumn{5}{|c|}{ Dias após Aplicação } \\
\hline & & 7 & 14 & 21 & 42 & Pré-colheita \\
\hline Testemunha sem capina & - & $0 \mathrm{c}^{1}$ & $0 \mathrm{~b}^{1}$ & $0 \mathrm{c}^{1}$ & $0 \mathrm{~b}^{1}$ & $0 \mathrm{~b}^{1}$ \\
\hline Testemunha capinada & - & $100 \mathrm{a}$ & $100 \mathrm{a}$ & $100 \mathrm{a}$ & $100 \mathrm{a}$ & $100 \mathrm{a}$ \\
\hline Imazethapyr+chlorimuron & $50,0+10,0$ & $100 \mathrm{a}$ & $100 \mathrm{a}$ & $100 \mathrm{a}$ & $100 \mathrm{a}$ & $100 \mathrm{a}$ \\
\hline Imazethapyr+chlorimuron & $50,0+12,5$ & $100 \mathrm{a}$ & $100 \mathrm{a}$ & $100 \mathrm{a}$ & $100 \mathrm{a}$ & $100 \mathrm{a}$ \\
\hline Imazethapyr+chlorimuron & $60,0+10,0$ & $100 \mathrm{a}$ & $100 \mathrm{a}$ & $100 \mathrm{a}$ & $100 \mathrm{a}$ & $100 \mathrm{a}$ \\
\hline Imazethapyr+lactofen & $50,0+96,0$ & $99 \mathrm{ab}$ & $97 \mathrm{a}$ & $96 \mathrm{ab}$ & $98 \mathrm{a}$ & $98 \mathrm{a}$ \\
\hline Imazethapyr+Lactofen & $50,0+120,0$ & $94 \mathrm{ab}$ & $96 \mathrm{a}$ & $99 \mathrm{ab}$ & $100 \mathrm{a}$ & $100 \mathrm{a}$ \\
\hline Imazethapyr+lactofen & $60,0+96,0$ & $96 \mathrm{ab}$ & 96 a & $94 \mathrm{~b}$ & $97 \mathrm{a}$ & 95 a \\
\hline Imazethapyr+lactofen & $60,0+120,0$ & $99 \mathrm{ab}$ & 99 a & $100 \mathrm{a}$ & $100 \mathrm{a}$ & $100 \mathrm{a}$ \\
\hline Imazethapyr+oxasulfuron & $50,0+37,5$ & $100 \mathrm{a}$ & $100 \mathrm{a}$ & $100 \mathrm{a}$ & $100 \mathrm{a}$ & $100 \mathrm{a}$ \\
\hline Imazethapyr+oxasulfuron & $60,0+37,5$ & $97 \mathrm{ab}$ & $100 \mathrm{a}$ & $100 \mathrm{a}$ & $100 \mathrm{a}$ & $100 \mathrm{a}$ \\
\hline Oxasulfuron & 45,0 & $99 \mathrm{ab}$ & $100 \mathrm{ab}$ & $100 \mathrm{ab}$ & $100 \mathrm{a}$ & $100 \mathrm{a}$ \\
\hline Chlorimuron+lactofen & $12,5+120,0$ & $100 \mathrm{a}$ & $100 \mathrm{ab}$ & $100 \mathrm{ab}$ & $100 \mathrm{a}$ & $100 \mathrm{a}$ \\
\hline Imazethapyr & 100,0 & $93 \mathrm{~b}$ & 96 a & $97 \mathrm{ab}$ & $100 \mathrm{a}$ & $100 \mathrm{a}$ \\
\hline Chlorimuron & 15,0 & $100 \mathrm{a}$ & $100 \mathrm{a}$ & $100 \mathrm{ab}$ & $100 \mathrm{a}$ & $100 \mathrm{a}$ \\
\hline Lactofen & 168,0 & $100 \mathrm{a}$ & 99 a & $99 \mathrm{ab}$ & $100 \mathrm{a}$ & $100 \mathrm{a}$ \\
\hline $\mathrm{F}$ & & $387,2^{*}$ & $836,7^{*}$ & $480,4^{*}$ & $1387,4^{*}$ & $350,2^{*}$ \\
\hline C.V. $(\%)$ & & 2,7 & 1,8 & 2,4 & 1,4 & 2,8 \\
\hline D.M.S. $(5 \%)$ & & 6,4 & 4,4 & 5,8 & 3,4 & 6,8 \\
\hline
\end{tabular}

'Médias seguidas da mesma letra de cada coluna, não diferem significativamente entre si. 
Tabela 3. Efeitos dos tratamentos no controle de Euphorbia heterophylla, na cultura da soja. Campo Mourão-PR, 1996/97.

\begin{tabular}{|c|c|c|c|c|c|c|}
\hline \multirow{3}{*}{ Tratamento } & \multirow{3}{*}{$\begin{array}{l}\text { Dose } \\
\text { (g/ha) }\end{array}$} & \multicolumn{5}{|c|}{ Controle (\%) } \\
\hline & & \multicolumn{5}{|c|}{ Dias após Aplicação } \\
\hline & & 7 & 14 & 21 & 42 & Pré-colheita \\
\hline Testemunha sem capina & - & $0 c^{1}$ & $0 \mathrm{~d}^{1}$ & $0 \mathrm{e}^{1}$ & $0 \mathrm{e}^{1}$ & $0 c^{1}$ \\
\hline Testemunha capinada & - & $100 \mathrm{a}$ & $100 \mathrm{a}$ & $100 \mathrm{a}$ & $100 \mathrm{a}$ & $100 \mathrm{a}$ \\
\hline Imazethapyr+chlorimuron & $50,0+10,0$ & $31 \mathrm{~b}$ & $92 \mathrm{a}$ & $96 \mathrm{a}$ & $95 \mathrm{a}$ & $100 \mathrm{a}$ \\
\hline Imazethapyr+chlorimuron & $50,0+12,5$ & $39 \mathrm{~b}$ & $90 \mathrm{a}$ & $96 \mathrm{a}$ & $95 \mathrm{a}$ & $99 \mathrm{a}$ \\
\hline Imazethapyr+chlorimuron & $60,0+10,0$ & $35 \mathrm{~b}$ & $86 \mathrm{ab}$ & $92 \mathrm{a}$ & $96 \mathrm{a}$ & $100 \mathrm{a}$ \\
\hline Imazethapyr+lactofen & $50,0+96,0$ & $97 \mathrm{a}$ & $95 \mathrm{a}$ & $93 \mathrm{a}$ & 93 a & $98 \mathrm{a}$ \\
\hline Imazethapyr+lactofen & $50,0+120,0$ & $97 \mathrm{a}$ & $91 \mathrm{a}$ & $93 \mathrm{a}$ & $90 \mathrm{ab}$ & $97 \mathrm{a}$ \\
\hline Imazethapyr+lactofen & $60,0+96,0$ & $99 \mathrm{a}$ & $96 \mathrm{a}$ & $94 \mathrm{a}$ & $93 \mathrm{a}$ & $97 \mathrm{a}$ \\
\hline Imazethapyr+lactofen & $60,0+120,0$ & $97 \mathrm{a}$ & $96 \mathrm{a}$ & 93 a & $96 \mathrm{a}$ & $99 \mathrm{a}$ \\
\hline Imazethapyr+oxasulfuron & $50,0+37,5$ & $21 \mathrm{bc}$ & $85 \mathrm{ab}$ & $87 \mathrm{ab}$ & $91 \mathrm{a}$ & $99 \mathrm{a}$ \\
\hline Imazethapyr+oxasulfuron & $60,0+37,5$ & $20 \mathrm{bc}$ & $87 \mathrm{ab}$ & $94 \mathrm{a}$ & $94 \mathrm{a}$ & $100 \mathrm{a}$ \\
\hline Oxasulfuron & 45,0 & $19 \mathrm{bc}$ & $70 \mathrm{bc}$ & $63 \mathrm{~cd}$ & $66 \mathrm{~cd}$ & $77 \mathrm{ab}$ \\
\hline Chlorimuron+lactofen & $12,5+120,0$ & $90 \mathrm{a}$ & $88 \mathrm{a}$ & $73 \mathrm{bc}$ & $74 \mathrm{c}$ & $69 \mathrm{~b}$ \\
\hline Imazethapyr & 100,0 & $32 \mathrm{~b}$ & $94 \mathrm{a}$ & $95 \mathrm{a}$ & $97 \mathrm{a}$ & $100 \mathrm{a}$ \\
\hline Chlorimuron & 15,0 & $26 \mathrm{~b}$ & $60 \mathrm{c}$ & $55 \mathrm{~d}$ & $58 \mathrm{~d}$ & $57 \mathrm{~b}$ \\
\hline Lactofen & 168,0 & $98 \mathrm{a}$ & $9 \mathrm{a}$ & $74 \mathrm{bc}$ & $77 \mathrm{bc}$ & $70 \mathrm{~b}$ \\
\hline $\mathrm{F}$ & & $65,5^{*}$ & $48,3 *$ & $71,2 *$ & $90,4^{*}$ & $26,5^{*}$ \\
\hline C.V. $(\%)$ & & 16,6 & 8,4 & 7,4 & 6,4 & 12,2 \\
\hline D.M.S. $(5 \%)$ & & 24,1 & 17,9 & 15,4 & 13,6 & 26,7 \\
\hline
\end{tabular}

${ }^{1}$ Médias seguidas da mesma letra dentro de cada coluna, não diferem significativamente entre si.

mente utilizados como lactofen, imazethapyr + lactofen e chlorimuron-ethyl + lactofen. A partir dos 14 D.A.A. até a pré-colheita o imazethapyr, isolado e em diferentes misturas e doses, controlou muito bem o amendoim-bravo, resultando numa eficiência mínima de $97 \%$, e diferindo significativamente dos tratamentos convencionais com lactofen, chlorimuron e chlorimuron + lactofen, os quais apresentaram uma eficiência inferior. Convém ressaltar que o amendoim-bravo estava com 4 a 5 folhas quando da aplicação dos produtos e que, normalmente, os tratamentos convencionais utilizados são recomendados para aplicação no estádio de 2 a 4 folhas definitivas. O oxasulfuron não diferiu dos melhores tratamentos na pré-colheita, mas foi inferior aos 21 D.A.A. e 42 D.A.A. Além disso, o controle de $77 \%$, obtido na précolheita, é considerado apenas regular, o que requer cuidados no uso deste herbicida em condições de média a alta infestação de E. heterophylla. Observou-se, ainda, um efeito residual do imazethapyr sobre a nova infestação de amendoim-bravo, e isto ocorreu tanto com o produto isolado como em mistura, sendo que os sintomas de fitotoxicidade nas plantas emergidas após a aplicação do produto foram observados até 21 D.A.A. Os demais herbicidas, com exceção do lactofen, também mostraram certo efeito residual só que com menor intensidade quando comparados ao imazethapyr.
Uma análise conjunta das Tabelas 2 e 3 mostra que não houve diferença significativa entre as diferentes misturas do imazethapyr com os outros latifolicidas no controle de B. pilosa e E heterophylla e que as misturas de imazethapyr, assim como o produto isolado, foram superiores ao chlorimuron-ethyl + lactofen $(12,5+120 \mathrm{~g} / \mathrm{ha})$, chlorimuronethyl isolado (15 g/ha), lactofen isolado (168 g/ha) e oxasulfuron isolado ( $45 \mathrm{~g} / \mathrm{ha}$ ) no controle de amendoim-bravo. Assim, neste caso, uma decisão de qual mistura deverá ser utilizada dependerá de uma análise de custos, uma vez que, tecnicamente, todas as misturas testadas com imazethapyr foram eficientes e não diferiram significativamente entre si.

Verificou-se que todos os tratamentos com herbicidas resultaram em injúrias nas plantas de soja (Tabela 4). Os sintomas observados incluíram amarelecimento, encarquilhamento e, nos casos mais severos, necroses nas folhas, como ocorreu nas misturas de imazethapyr + lactofen, chlorimuron-ethyl + lactofen e lactofen isolado. A partir dos 14 D.A.A. todos os sintomas descritos sumiram e não se observou qualquer espécie de injúria visual nas partes novas das plantas. Todos os herbicidas, isolados ou em misturas, retardaram inicialmente o desenvolvimento da soja, provo- 
Tabela 4. Fitotoxicidade (escala E.W.R.C.), número de plantas / $3 \mathrm{~m}$ na pré-colheita e produtividade da soja submetida aos diversos tratamentos. Campo Mourão, PR, 1996/97.

\begin{tabular}{|c|c|c|c|c|c|c|c|}
\hline \multirow{3}{*}{ Tratamento } & \multirow{3}{*}{$\begin{array}{l}\text { Dose } \\
\text { (g/ha) }\end{array}$} & \multirow{2}{*}{\multicolumn{4}{|c|}{$\begin{array}{c}\begin{array}{c}\text { Fitotoxicidade } \\
\text { (Escala E.W.R.C.) }\end{array} \\
\text { Dias após Aplicação }\end{array}$}} & \multirow{3}{*}{$\begin{array}{l}\text { Pré-colheita } \\
\text { (plantas/3m) }\end{array}$} & \multirow{3}{*}{$\begin{array}{l}\text { Produtividade } \\
\quad \text { (kg/ha) }\end{array}$} \\
\hline & & & & & & & \\
\hline & & 7 & 14 & 21 & 42 & & \\
\hline Testemunha sem capina & - & 1,0 & 1,0 & 1,0 & 1,0 & $24 b^{1}$ & $1039 b^{1}$ \\
\hline Testemunha capinada & - & 1,0 & 1,0 & 1,0 & 1,0 & $36 \mathrm{ab}$ & 3296 a \\
\hline Imazethapyr+chlorimuron & $50,0+10,0$ & 4,5 & 1,0 & 1,0 & 1,0 & $38 \mathrm{a}$ & $2944 \mathrm{a}$ \\
\hline Imazethapyr+chlorimuron & $50,0+12,5$ & 4,5 & 1,0 & 1,0 & 1,0 & $36 a b$ & $2972 \mathrm{a}$ \\
\hline Imazethapyr+chlorimuron & $60,0+10,0$ & 4,5 & 1,0 & 1,0 & 1,0 & $38 \mathrm{a}$ & $2861 \mathrm{a}$ \\
\hline Imazethapyr+lactofen & $50,0+96,0$ & 5,0 & 1,0 & 1,0 & 1,0 & $38 \mathrm{a}$ & $2907 a$ \\
\hline Imazethapyr+lactofen & $50,0+120,0$ & 5,0 & 1,0 & 1,0 & 1,0 & $34 \mathrm{ab}$ & $3130 \mathrm{a}$ \\
\hline Imazethapyr+lactofen & $60,0+96,0$ & 5,0 & 1,0 & 1,0 & 1,0 & $37 \mathrm{ab}$ & $3055 \mathrm{a}$ \\
\hline Imazethapyr+lactofen & $60,0+120,0$ & 5,0 & 1,0 & 1,0 & 1,0 & $40 \mathrm{a}$ & $3157 \mathrm{a}$ \\
\hline Imazethapyr+oxasulfuron & $50,0+37,5$ & 4,0 & 1,0 & 1,0 & 1,0 & $43 \mathrm{a}$ & $3027 \mathrm{a}$ \\
\hline Imazethapyr+oxasulfuron & $60,0+37,5$ & 4,0 & 1,0 & 1,0 & 1,0 & $40 \mathrm{a}$ & $3055 \mathrm{a}$ \\
\hline Oxasulfuron & 45,0 & 4,0 & 1,0 & 1,0 & 1,0 & $36 \mathrm{ab}$ & $2490 \mathrm{a}$ \\
\hline Chlorimuron+lactofen & $12,5+120,0$ & 5,0 & 1,0 & 1,0 & 1,0 & $34 \mathrm{ab}$ & $2500 \mathrm{a}$ \\
\hline Imazethapyr & 100,0 & 4,0 & 1,0 & 1,0 & 1,0 & $41 \mathrm{a}$ & $3139 a$ \\
\hline Chlorimuron & 15,0 & 4,0 & 1,0 & 1,0 & 1,0 & $39 \mathrm{a}$ & $2555 \mathrm{a}$ \\
\hline Lactofen & 168,0 & 5,0 & 1,0 & 1,0 & 1,0 & $37 \mathrm{ab}$ & $2620 \mathrm{a}$ \\
\hline $\mathrm{F}$ & & - & - & - & - & $2,6^{*}$ & $4,8^{*}$ \\
\hline C.V. (\%) & & - & - & - & - & 13,8 & 17,2 \\
\hline D.M.S. (5\%) & & - & - & - & - & 13,1 & 1237,2 \\
\hline
\end{tabular}

'Médias seguidas da mesma letra dentro de cada coluna, não diferem significativamente entre si.

cando redução do porte da cultura quando comparados á testemunha capinada. Estes sintomas persistiram até os 21 D.A.A., sendo que a partir da avaliação feita aos 42 D.A.A. a soja se recuperou nos tratamentos com herbicidas, igualando-se à testemunha capinada. Nem o estande nem a produtividade foram afetados pelos herbicidas citados (Tabela 4). Com relação à produtividade, a interferência das plantas daninhas sobre a cultura da soja resultou numa queda média de $64 \%$, o que ratifica as observações de Karan et al. (1993a; 1993b), Spadotto \& Luchiari (1993) e Rodrigues \& Pitelli (1994).

\section{CONCLUSÕES}

As misturas de tanque de imazethapyr + chlorimuronethyl $(50+10,50+12,5$ e $60+10 \mathrm{~g} / \mathrm{ha})$, imazethapyr + lactofen $(50+96,50+120,60+96$ e $60+120 \mathrm{~g} / \mathrm{ha})$ e imazethapyr + oxasulfuron $(50+37,5$ e $60+37,5 \mathrm{~g} / \mathrm{ha})$ foram muito eficazes no controle de Bidens pilosa e Euphorbia heterophylla, resultando num mínimo de $97 \%$ de eficiência na fase de précolheita.
Não houve diferença significativa entre as misturas em tanque contendo imazethapyr.

As misturas de tanque contendo imazethapyr foram superiores ao chlorimuron-ethyl + lactofen, chlorimuron-ethyl isolado e lactofen isolado, para o controle de Euphorbia heterophylla,

Nenhuma mistura de tanque de imazethapyr com outros herbicidas causou fitotoxicidade que comprometesse o estande ou produtividade da cultura.

\section{LITERATURA CITADA}

AGRIANUAL. Anuário estatístico da agricultura - FNP Consultoria e Comércio, ARGOS, São Paulo, SP, v.1 p.471-473, 1999.

EMBRAPA- CNPSo. Recomendaçôes técnicas para a cultura da soja no Paraná 1996/1997. Londrina; EmbrapaSoja, 1996. 1 87p. (Embrapa-Soja. Documentos,97). 
GAZZIERO, D.L.P.; KIIHL, R.A.S.; ALMEIDA, L.A.; BRIGIIENTI, A. M.; VOLL, E.; MACIEL, C.D.G. Avaliação de herbicidas do grupo químico das imidazolinonas, aplicados em pós-emergência, em soja tolerante. In: CONGRESSO BRASILEIRO DE SOJA, 1; 1999, Londrina. Anais.. Londrina: EMBRAPA-CNPSoja, 1999. p.398.

KARAN, D.; GAZZIERO, D.L.P.; VOLL, E.; MALASSEN, M.C. Estudo da interferência de plantas daninhas com a cultura da soja (Glycine max L. Merril) em casa de vegetação. In: CONGRESSO BRASILEIRO DE HERBICIDAS E PLANTAS DANINHAS, 19, 1993, Londrina. Resumos... Londrina: SBHED, 1993a. p.7374.

KARAN, D.; VOLL, E.; GAZZIERO, D.L.P.; CAÇÃO, L.E.F. Estudo da interferência de plantas daninhas com a cultura da soja (Glycine max L. Merril). In: CONGRESSO BRASILEIRO DE IJERBICIDAS E PLANTAS DANINHAS, 19, 1993, Londrina. Resumos... Londrina: SBHED, 1993b. p.32-33.

LACA-BUENDIA, J.P.; RAFAEL, J.O.V. Avaliação de herbicidas em diferentes modos e épocas de aplicação, para o controle de plantas daninhas, na cultura da soja. In: MELHORANÇA, A.L. Reunião de Pesquisadores em Controle de Plantas Daninhas nos Cerrados, 12., 1999, Corumbá, MS. Controle químico de plantas daninhas nos cerrados. Dourados: EMBRAPA Agropecuária Oeste, 1999a. p.31 -38. (EMBRAPA Agropecuária Oeste. Documentos, 3 ).

LACA-BUENDIA, J.P.; RAFAEL, J.O.V. Avaliação de herbicidas em pósemergência, para o controle de plantas daninhas na cultura da soja. In: MELHORANÇA, A.L. Reunião de Pesquisadores em Controle de Plantas Daninhas nos Cerrados, 12, 1999, Corumba, MS. Controle químico de plantas daninhas nos cerrados. Dourados: EMBRAPA Agropecuária Oeste, 1999b. p.38-50. (EMBRAPA Agropecuária Oeste. Documentos, 3$)$.
LORENZI, H. Plantas daninhas do Brasil. Terrestres, aquáticas, parasitas, tóxicas e medicinais. 2a ed. Nova Odessa: Plantarum. $1991.440 \mathrm{p}$

MELHORANÇA, A.L. Estudo da eficiência de metribuzin+sulfentrazone no controle das plantas daninhas na cultura da soja. In: MELHORANÇA, A.L. Reunião de Pesquisadores em Controle de Plantas Daninhas nos Cerrados, 12., 1999, Corumbá, MS. Controle químico de plantas daninhas nos cerrados. Dourados: EMBRAPA Agropecuária Oeste, 1999. p.67-70. (EMBRAPA Agropecuária Oeste. Documentos, 3).

MELHORANÇA, A.L.; PEREIRA, F.A.R. Eficiência do herbicida lactofen no controle de Euphorbia heterophylla, resistente aos herbicidas inibidores da enzima acetolactato sintase (ALS). In: MELHORANÇA, A.L. Reunião de Pesquisadores em Controle de Plantas Daninhas nos Cerrados, 12., 1999, Corumbá, MS. Controle químico de plantas daninhas nos cerrados. Dourados: EMBRAPA Agropecuária Oeste, 1999. p.1 1-14. (EMBRAPA Agropecuária Oeste. Documentos, 3).

RODRIGUES, B.N.; PITELLI, R.A. Quebra de dormência em sementes de Commelina benghalensis. Planta Daninha, v. 12, n.2, p.1 06-110, 1994.

SPADOTTO, C.A., LUCHIARI, F. Índice de competitividade de espécies de plantas daninhas com a soja. In: CONGRESSO BRASILEIRO DE HERBICIDAS E PLANTAS DANINHAS, 19, 1993, Londrina. Resumos... Londrina: SBHED, 1993. p.32-33.

VARGAS, L.; SILVA, A.A.; BOPEM, A.; PEZENDE, S.T.; FERREIRA, F.A.; SEDIYAMA, T. Resistência de plantas daninhas a herbicidas. Viçosa, MG: UFV, $1999.131 \mathrm{p}$.

VIDAL, R.A. Sistema integrado de controle de Euphorbia heterophy/ia em soja. In: CONGRESSO BRASILEIRO DE SOJA, 1, 1999, Londrina. Anais... Londrina: EMBRAPA-CNPSoja, 1999. p.421. 